\title{
AVANT - PROPOS
}

Le changement du titre de cette deuxième édition de l'ouvrage "Endocrinologie, fondements physiologiques", qui devient "Endocrinologie et communications cellulaires", traduit l'ampleur des modifications ou des compléments apportés à la première édition.

La contribution des collaborateurs, qui ont bien voulu apporter le fruit de leur compétence à cet ouvrage, a permis non seulement une réactualisation des données de l'Endocrinologie, mais aussi une extension du public concerné.

Les collaborateurs en médecine clinique (Docteurs Robert ELKAÏM, Philippe LÉGER et Danielle PALLO) et hospitalo-universitaires (Professeurs Ivan BACHELOT et Robert HALIMI) ont contribué à une "médicalisation" de cet ouvrage et en font un outil utilisable non seulement pour les étudiants en médecine, mais aussi pour les médecins désireux d'actualiser leurs connaissances en endocrinologie. La participation de spécialistes de la communication cellulaire, comme Claude COCHET et Jean VERDETTI, devrait faire de cet ouvrage une référence pour les étudiants de troisième cycle orientés vers la recherche dans ce domaine.

Compte tenu de son importance croissante dans la vie quotidienne, le stress a été particulièrement développé (chap. $6, \S 5$ ), ainsi que ses conséquences sur le système immunitaire (chap. 16, § 4).

La partie "Endocrinologie de la reproduction" a été enrichie d'un chapitre (chap. 15) qui apporte des données actuelles particulièrement utiles aux enseignants du secondaire, dans le cadre du planning familial et de la procréation médicalement assistée.

L'évolution du profil démographique vers un accroissement du nombre de personnes âgées a justifié l'approfondissement du rôle de l'épiphyse en rapport avec le vieillissement (chap. 10, § 2.3) et des additions dans d'autres chapitres sur les aspects endocriniens du vieillissement (chap. $4, \S 4.2 .6$ et $4.3 .3 ; 6, \S 6$ ).

Dans cette deuxième édition, nous avons voulu également souligner le développement de disciplines intégrées qui donnent lieu à des recherches fécondes, telles la psycho-neuro-endocrinologie (chap. $4, \S 10$ ), l'endocrino-immunologie (chap. 16) et la psycho-neuro-immunologie (chap.16, § 4) dont l'ensemble constitue le cadre d'une psycho-neuro-immuno-endocrinologie.

Enfin, cette actualisation n'a été possible que grâce au Professeur Jean VERDETTI qui a accepté d'être co-auteur de cette deuxième édition, en nous faisant bénéficier de ses connaissances, fondées sur une recherche de pointe, et de ses qualités de pédagogue. 
Nos remerciements vont à J. BORNAREL et à son équipe, pour leur patience et leur confiance malgré la charge représentée par cette deuxième édition, ainsi qu'aux membres du comité de lecture, J. ANDRÉ, E.E. BAUliEU, E. ChAmbAZ et J.E. DUMONT qui ont accepté de lire cet ouvrage et de nous exprimer leur avis, tout particulièrement E.E. BAULIEU et E. CHAMBAZ dont les remarques et critiques ont été très constructives. 